rotating ${ }^{3}$ about its chain axis independent in phase of other rotating groups, then the result given above would be obtained. If the temperature should be sufficiently lowered, this complete rotation would be replaced by slight oscillation about some equilibrium positions.

Observations were made on primary amylammonium chloride at approximately liquid air tempera.tures. The density, dotermined by susponsion in mixtures of liquid nitrogen and oxygen, is c. $1 \cdot 0$, probably a little greater than the value 0.953 at $25^{\circ} \mathrm{C}$. Diffraction lines on powder photographs (CuK radiation) at liquid air temperatures are similar in spacings to, but markedly different in intensities from, those at room temperatures; some few additional lines requiring a larger unit of structure are also present. It is difficult to dotermine accurately the structural characteristics of such a complex compound from powder photographs alone. The photographs at liquid air temperatures, however, indicate, from their similarity to photographs at $25^{\circ}$, that the crystals have approximately orthogonal axes and that the atomic arrangement in the unit of structure containing $4 \mathrm{NH}_{3} \mathrm{C}_{5} \mathrm{H}_{11} \mathrm{Cl}$, with the dimensions $a=b=7.0 \mathrm{~A}$., $c=c .16 .6 \mathrm{~A}$., is closely similar to that in the unit of structure previously described. The presenco of planes such as $(210)$ and $(300)$, referred to the axes of the larger unit of structure, could be explained by alteration in the structure but probably is best accounted for by absence of the suspected molecular rotation that leads to the fortuitous determination of the unit of structure and atomic arrangement at room tomperatures.

The best test for a possible collinear arrangement of the carbon atoms of $a \mathrm{C}_{5} \mathrm{II}_{11}$ group is the absence of reflections in odd orders from planes $(h k 0)$ with $(h+k)$ odd. (Indices referred to a unit of structure having $a=b=5.0 \mathrm{~A} ., c=16.6 \mathrm{~A}$.). The large axial ratio makes it difficult to distinguish betwoon reflections from $(h k 0)$ and $(h k l)$ with $l$ unity on powder photographs. Reflections from (200) and (201), however, are very weak at liquid air temperatures, while reflections from (200) are strong at room temperature. This change in the intensity of (200) could be explained by a groat departure of the chain axes of tho $\mathrm{C}_{5} \mathrm{H}_{11}$ groups from parallelism with the tetragonal axis of the crystal at liquid air temperaturos, or, as is most probable, by a departure of the carbon atoms from a collinear arrangement.

The most immediate conclusion is that the carbon atoms in a $\mathrm{C}_{5} \mathrm{H}_{11}$ group are arranged in a 'zig-zag' manner and that the characteristics of the X-ray diffraction photographs made at room temperatures from crystals of primary amyl ammonium chloride arise partially from rotation of the $\mathrm{C}_{5} \mathrm{H}_{11}$ groups about their chain axes. The configuration of a hydrocarbon chain as deduced from the crystal structure of the primary alkyl ammonium halides is thus probably the same as that first found by Müller and Shearer ${ }^{4}$ for some long chain aliphatic compounds. The carbon to carbon separation along the chain axis is $c$. $1 \cdot 20-1 \cdot 30 \mathrm{~A}$, and the carbon-carbon distanco might well bo c. 1.54 A.

It thus seems, as Pauling has indicated, that in a crystal containing molecules or molecular groups with small moments of inertia about some axes, these molecules may undergo rotational motion about these axes.

Sterding B. Heindrioks.

Fertilizer and Fixed Nitrogen Investigations, Bureau of Chemistry and Soils, Washington, D.C.

' S. B. Hendricks, Z. f. Krist., 67, 106, 475; 1928 ; 68, 189; 1928. S. B. Henricks, Z. f. Krist. (In press.)

3 Sce Linus Pauling, Phys. Rev., July 1930.

Jour. Chem. Soc, 123, 3156; 1923. G. Shearer, Proc. Roy Soc. A. 108, 655; 1925 .

No. 3170, VoL. 126]

\section{Raman Effect, Fluorescence and Colour of Diamonds.}

THE Raman spectra of various simple substances, including especially the non-metallic elements such as phosphorus, chlorine, and carbon, have been investigated by me. In the course of this work numerous samples of diamond were examined, and a brief roport of the results may be of interest as supplementing the accounts which have already appeared in NATURF of May 10.

The extreme sharpness of the Rarnan lines observed with diamond invited attempts to measure their wavelength with all possible precision. Seven different diamonds gave results identical within the limits of error of measurement. The best representative value for the infra-red wave-number was found to be $1331.5 \pm$ $0.5 \mathrm{~cm}^{-1}$, in agreement with Ramaswamy's measurements but differing rather seriously from the value 1342 given by Robertson and Fox. In the case of

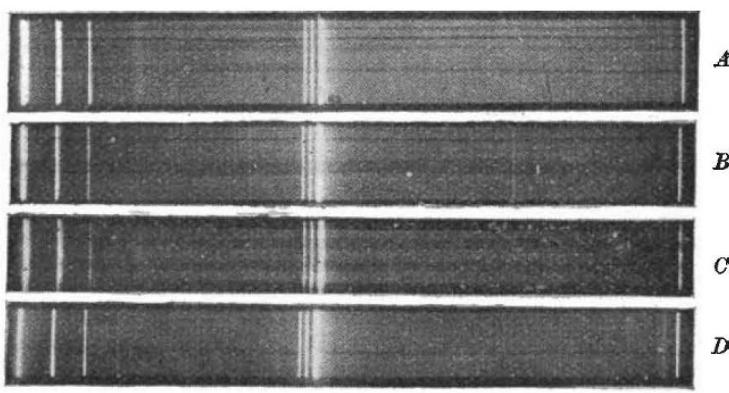

Fia. 1.

one very imperfect diamond, it was noticod that the line was diffuse and distorted and was accompanied by a faint companion line on the longer wave-length side.

A feature of importance not mentioned in NATURE of May 10 is the continuous spectrum accompanying the Raman lines. This appears with a fairly well defined edge on the violet side at about $\lambda 4240 \mathrm{~A}$., and stretches out towards the visible region. Immediately preceding it are two bands of which the first at 4152 to $4162 \mathrm{~A}$. is the more intense. The intensity of the bands and of the continuous spectrum varies in a remarkable way with the colour of the diamond. 'They are specially conspicuous with diamonds of a pale blue colour, and are extremely feeble with white diamonds. Per contra, the Raman lines are very difficult to observe with blue diamonds, and are most easily obtained with colourless diamonds.

In Fig. 1, $A$ taken with a blue diamond and $B$ with a white diamond exhibit these features. $C$ was taken with a diamond, having the palest yellow tinge and showed both the Raman lines and the bands accompanicd by a continuous spectrum of considerable intensity. From the fact that the introduction of a filter cutting out the mercury line at $4046 \mathrm{~A}$. eliminates the bands and practically the whole of the continuous spectrum, it may be inferred that these represent fluorescence of a special type. The aggregate intonsity of the continuous spectrum appearing in $A$ must have been very considerable, and it was thought that the blue colour of the diamond in ordinary daylight was really due to this fluorescent radiation. $D$ was obtained with another diamond of a strikingly yellow colour. It gave feeble Raman lines, and a continuous spectrum (without bands) stretching practically over the whole region from $3800 \mathrm{~A}$. to the red end.

S. Bhaga Vantam.

210 Bowbazar Street,

Calcutta, India, June 14. 